\title{
Single Incision Transumbilical Laparoscopic Roux-en-Y Gastric Bypass-Our Technique
}

\author{
Jasmeet Singh Ahluwalia $\cdot$ Po-Chih Chang • \\ Lee-Ren Yeh • Hung-Yu Lin • Shu-Ching Chi • \\ Chih-Kun Huang
}

Published online: 11 July 2014

(C) Springer Science+Business Media New York 2014

\begin{abstract}
Laparoscopic Roux-en-Y gastric bypass (LRYGB) is considered the gold standard procedure in bariatric surgery but requires 4-7 ports. We have reported the first single incision transumbilical Roux-en-Y gastric bypass (SITU-RYGB) in 2009 (Huang et al. Obes Surg 19:1711-1715, 2009). Over the years, we have standardized our procedure and this video highlights the same by showing both inside and outside views. This video was shot from outside as well to give better understanding of the procedure. A $4.5-\mathrm{cm}$ incision was made according to the contour of umbilicus and space was created over the sheath to give more range of movement to the instruments. The procedure was carried out using conventional laparoscopic instruments and replicating all the steps of the procedure under adequate visualization. Picture-inpicture effect has been used at important steps. Findings were recorded. The procedure took $96 \mathrm{~min}$ without any intraoperative complication. Blood loss was $20 \mathrm{cc}$. The incision was hardly noticeable at the end of the procedure. We have previously compared our results of SITU-RYGB with that of our multiport RYGB where operative time was longer for SITU-RYGB versus multiport technique (101.1 vs. $81.1 \mathrm{~min}, P=0.001$ ) (Huang et al. Surg Obes Relat Dis 8:201-207, 2012). No difference in
\end{abstract}

Electronic supplementary material The online version of this article (doi:10.1007/s11695-014-1318-7) contains supplementary material, which is available to authorized users.

J. S. Ahluwalia $\cdot$ P.-C. Chang $\cdot$ C.-K. Huang $(\bowtie)$

Bariatric and Metabolic International (BMI) Surgery Center, E-Da

Hospital, 1, E-Da Road, Kaohsiung City 82445, Taiwan

e-mail: dr.ckhuang@hotmail.com

L.-R. Yeh

Department of Radiology, E-Da Hospital, Kaohsiung City, Taiwan

H.-Y. Lin

Department of Urology, E-Da Hospital, Kaohsiung City, Taiwan

S.-C. Chi

Department of Nursing, E-Da Hospital, Kaohsiung City, Taiwan complications was observed. The SITU-LRYGB patients reported greater satisfaction related to scarring than those who had undergone five-port surgery $(P=0.005)$. Difference in analgesia requirement was not statistically significant. There was no mortality. Compared with conventional LRYGB, SITU-RYGB resulted in acceptable complications, the same recovery, comparative weight loss, and better patient satisfaction related to scarring.

Keywords Single-incision RYGB · Single-incision bariatric surgery $\cdot$ Bariatric $\cdot$ RYGB $\cdot$ SILS $\cdot$ SITU RYGB

Conflict of Interest The authors have no conflict of interest.

Funding There is no external source of funding.

\section{References}

1. Huang CK, Houng JY, Chiang CJ, et al. Single-incision transumbilical laparoscopic Roux-en-Y gastric bypass: a first case report. Obes Surg. 2009;19:1711-5.

2. Huang CK, Lo CH, Houng JY, et al. Surg Obes Relat Dis. 2012;8:201-7. 